\title{
Hacia una apuesta etnográfica para la glotopolítica
}

Virginia Zavala

Recebido em: 30 de janeiro de 2020

Aceito em: 6 de março de 2020
Virginia Zavala es Ph.D en Sociolingüística por la Universidad de Georgetown en Estados Unidos y profesora principal de la Pontificia Universidad Católica del Perú en la especialidad de Lingüística. Sus investigaciones abordan la problemática del lenguaje y la educación, con un foco en el quechua, y desde una perspectiva etnográfica, interdisciplinaria y de análisis del discurso. Pontificia Universidad Católica del Perú

Contato: vzavala@pucp.edu.pe Peru 
PALABRAS CLAVE: slotopolítica; etnografía; análisis textual, perspectiva crítica

KEYWORDS: slotopolitics; ethnography; textual analysis; critical perspective
Resumen: Este artículo argumenta que la perspectiva histórica y crítica adoptada por la slotopolítica podría enriquecerse con el enfoque etnográfico. A partir del involucramiento sostenido del investigador a lo largo de un tiempo prolongado en un lugar (o lugares) específicos y la combinación -y contraste- de varias fuentes de información y observación, la etnografía permite situar los textos en su contexto de producción y comprender mejor qué es lo que estos "hacen" a nivel de las prácticas sociales. Desde una conceptualización de la etnografía como método, metodología y teorización profunda, se ejemplificará lo anterior a partir de una disputa por la representación del quechua entre dos tipos de comunidades de práctica involucradas en el desarrollo de políticas lingüísticas en el Perú. Se sostendrá que la "mirada interna" que consigue la etnografía permite explicar mejor los procesos implicados en la construcción de ideologías lingüísticas.

Abstract: This article argues that the historical and critical perspective adopted by slotopolitics would be enriched by the ethnosraphic approach. Based on the sustained involvement of the researcher over a long period of time in a specific place (or places) and the combination - and contrast - of various sources of information and observation, ethnography makes it possible to situate texts in their contexts of production and to better understand what they "do" at the level of social practices. From a conceptualization of ethnography as a method, methodology and deep theorizing, the above will be exemplified with a dispute over the representation of Quechua between two types of communities of practice involved in the development of language policies in Peru. It will be argued that the "internal perspective" achieved by ethnography allows for a better explanation of the processes involved in the construction of language ideologies. 


\section{INTRODUCCIÓN}

Desde hace varias décadas, el estudio social del lenguaje se ha ido distanciando de una perspectiva más descriptiva y positivista para abordarlo desde la noción de práctica social y siempre en vínculo con el ejercicio del poder. La diferencia entre la sociolingüística crítica del norte global y la antropología lingüística norteamericana es cada vez más difícil de establecer, pues ambas corrientes muestran un gran número de puntos de convergencia en el estudio del lenguaje desde una mirada sociocultural (Bucholtz y Hall, 2008; Heller, Pietikainen y Pujolar; 2018). En el contexto hispano, la glotopolítica como perspectiva de estudio se suma a las anteriores corrientes para comprender la imbricación entre el lenguaje y la política, no solo en el marco de decisiones institucionales, sino también de los entornos sociales "en los que se tematiza el lenguaje en la construcción y negociación de un orden social donde está en juego la distribución del poder" (Del Valle, 2017, 22-23). Esta perspectiva glotopolítica se ha interesado por enriquecer el campo más clásico de las políticas lingüísticas desarrollado en los países del norte, a partir de un enfoque interdisciplinario, crítico e historiográfico. Como señala Del Valle (2017), la perspectiva histórica es la que permite ver la relación dinámica e inseparable entre el lenguaje y sus condiciones de producción.

En este trabajo, me gustaría argumentar que la perspectiva histórica adoptada por la mirada glotopolítica necesita de la etnográfica para situar los textos en su contexto de producción y para comprender mejor qué es lo que los textos "hacen" a nivel de las prácticas sociales. Sobre la base de una serie de trabajos etnográficos que he desarrollado a lo largo de mi 
carrera, analizaré las ideologías lingüísticas sobre el quechua por parte de dos comunidades de práctica (Lave y Wenger, 1991) involucradas en el desarrollo de políticas lingüísticas en el Perú, aunque me centraré más en una de ellas. Específicamente, discutiré cómo los "expertos" del quechua desde diferentes espacios institucionales construyen una versión del quechua y de lo quechua, y jóvenes activistas emergentes disputan esta manera de construir la realidad. Para poder entender bien las razones que subyacen a estos desacuerdos, no solo analizaré diferentes tipos de textos a partir de un esfuerzo contextualizador, sino que también echaré mano de entrevistas y conversaciones con los actores sociales que los produjeron, y de observaciones de las prácticas en las que éstos se utilizaron. La presentación de la información etnográfica no pretende ser exhaustiva, pues estará al servicio de la discusión teórica y metodológica.

\section{LeNGUAJE, PRÁCTICA SOCIAL Y PERSPECTIVA ETNOGRÁFICA}

Los estudios críticos del lenguaje, en los que se incluye la mirada glotopolítica, lo abordan como una práctica social que siempre tiene consecuencias en la conformación de la sociedad. Las prácticas del lenguaje equivalen a conjuntos de actividades que son centrales a la organización de la vida social, están ideológicamente definidas, y reflejan (y construyen) diferentes sentidos de vida social en el marco de relaciones de poder. Como lo recuerda Bourdieu (1997), las prácticas son acciones con una historia, que se encargan de articular el mundo material con el simbólico. Dicho de otra manera: 
la vida social está impregnada de prácticas de diferente tipo (educativas, religiosas, bancarias, recreativas, etc.) y las prácticas lingüísticas siempre forman parte de otras.

Sabemos que los textos son las instancias en las que se materializa el objeto de estudio de la glotopolítica (Del Valle, 2017). Sin embargo, como los textos siempre se desarrollan como parte de las prácticas sociales, el reto consiste en no perdernos en el análisis textual, sino en situarlo siempre en acciones particulares. Después de todo, los textos siempre se deben analizar como "discursos", en tanto huellas de un proceso de producción que involucra a grupos sociales particulares en condiciones históricas concretas (Arnoux y Nothstein, 2013). A diferencia de una serie de corrientes de análisis del discurso, la perspectiva glotopolítica no prioriza el análisis de las estructuras discursivas sino, más bien, la comprensión de los procesos históricos, esto con el objetivo de identificar las condiciones materiales que posibilitan o dificultan la producción de los discursos estudiados. En ese sentido, la glotopolítica estudia la materialidad del discurso en función de otros fenómenos que se buscan explicar, como el nacionalismo, el neocolonialismo o los procesos de integración regional, siempre en diálogo con disciplinas como la historia, los estudios culturales o la sociología de la literatura. No obstante, para tal fin, no solo resulta útil el análisis histórico, sino también la perspectiva etnográfica. El proyecto etnográfico consiste en describir e interpretar las prácticas que organizan la experiencia cotidiana de la gente desde las voces de los actores sociales involucrados. Como tal, nos desafía a que nuestra unidad de análisis ya no sean los textos sino las prácticas (donde emergen 
los textos) y nos ayuda precisamente a cerrar la brecha entre el análisis del texto y el análisis del contexto (Scollon y Scollon, 2004, Pennycook, 2010).

A contraposición de lo que a veces se suele creer, la etnografía no constituye solo un método específico, que implicaría, por ejemplo, hacer entrevistas a profundidad. Si bien la etnografía se asocia con métodos particulares, es importante situarla también como una metodología y como una teorización profunda de las acciones humanas (Lillis, 2008). Más allá de métodos puntuales, la etnografía como metodología supone el involucramiento sostenido del investigador a lo largo de un tiempo prolongado en un lugar (o lugares) específicos y la combinación (y contraste) de varias fuentes de información y observación. Esto último implica el uso de por lo menos dos métodos básicos: la observación (participante y no participante) de prácticas y la conversación informal con las personas. El objetivo del involucramiento prolongado y la combinación de diversos métodos es llegar a comprender los significados situados, complejos y dinámicos de las prácticas de los participantes desde una mirada émica o, en otras palabras, desde una mirada "interna” a los sujetos que participan en la investigación. Esto, a su vez, nos lleva a concebir la etnografía como una teorización profunda que ayuda a comprender la brecha ontológica entre texto y contexto. Desde esta conceptualización más amplia, la etnografía nos permite relacionar el análisis textual comprendido éticamente desde la mirada del investigador con las comprensiones informadas émicamente por los participantes del estudio en un contexto histórico particular. Así, una etnografía propone un argumento sobre un problema social en torno a cómo lo ven sus propios actores. 
La glotopolítica ha echado mano de la categoría teórica de las ideologías linguísticas "como elementos fundamentales en la identificación y análisis de los regímenes de la normatividad en las cuales necesariamente se interpretan las prácticas lingüísticas" (Arnoux y Del Valle 2010, 10). No obstante, si bien las ideologías lingüísticas pueden expresarse en diferentes tipos de textos, también es importante rastrear los procesos a partir de los cuales se solidifican. El enfoque etnográfico nos permite acercarnos -con un mayor poder explicativo- a estos procesos indexicales por medio de los cuales las formas lingüísticas van articulándose con tipos de personas, situaciones y contextos en el marco de valoraciones específicas (Irvine y Gal, 2000, Blommaert, 2006).

En suma, entonces, la mirada etnográfica nos da pistas para entender qué tipo de acciones desarrollan las personas con los textos, tanto en términos inmediatos como más ideológicos. Para tal fin, no solo es importante realizar un análisis contrastivo a nivel de los textos, sino entre los textos, las observaciones de las acciones donde se inscriben estos textos y las conversaciones con los actores sociales involucrados en la producción, distribución y consumo de los mismos. Esta triangulación, entre materiales pero también entre tipos de datos recogidos, me han ayudado a entender mejor las diferentes formas en las que emergen las representaciones del quechua y lo quechua a través de variados tipos de procesos; cómo estas representaciones están vinculadas a intereses o ideologías sociales, culturales, políticos o económicos particulares; qué tensiones existentes en la sociedad revelan; y cuáles son las consecuencias sobre cómo son posicionados diferentes tipos de hablantes. 


\section{LA DISPUTA POR EL OUECHUA}

\subsection{Los expertos del quechua en la construcción de diferencia y desigualdad}

Durante un periodo de trabajo etnográfico prolongado en varios espacios empecé a darme cuenta de que, dentro de la población de hablantes de quechua, se estaba erigiendo una comunidad de práctica de "expertos" del quechua quienes se estaban construyendo como hablantes autorizados y legítimos de la lengua para diferenciarse del resto. A través de un proceso de recursividad fractal (Irvine y Gal, 2000), que replicaba las relaciones de poder entre el castellano y las lenguas originarias, desarrollaban una distinción entre "los que saben quechua" y "los que no saben quechua" a partir de ideologías lingüísticas particulares. Este fenómeno muestra que la diferencia puede equivaler a desigualdad, no solo entre lo que llamamos "lenguas", sino también al interior de ellas (Blommaert, 2004), y que todo análisis de política lingüística debe tomar en cuenta estas dinámicas.

Daré un poco de contexto. Este grupo de quechuahablantes a los que estoy llamando "expertos" fueron socializados en la lengua originaria en comunidades rurales durante su primera infancia y, luego de pasar por la educación básica y estudiar para ser maestros de escuela, en la mayoría de los casos, ante la necesidad de implementar la educación intercultural bilingüe en el país durante las décadas de 1980 y 1990, fueron capacitados por lingüistas en diferentes tipos de programas. La formación de estos maestros se realizó desde una lingüística descriptiva poco crítica que tenía una visión de la lengua como un código delimitado y objetivado. Actualmente, estos “expertos” viven en ciudades, hablan principalmente español y trabajan como 
especialistas de Educación Intercultural Bilingüe en oficinas del Ministerio de Educación, enseñan quechua en universidades o laboran en ONGs que se ocupan de cuestiones educativas. Esta demanda creciente por el quechua se sitúa en un contexto en el que el discurso sobre los derechos lingüísticos sigue siendo fuerte, pero el quechua está surgiendo como un recurso económico que provee acceso a empleos y capital material (Del Percio et al., 2017). Por lo tanto, ahora tienen poder económico y simbólico, y representan una clase media quechua urbana educada.

Quisiera puntualizar que este fenómeno resulta nuevo con relación a lo que sucedía en la década de 1970 en el Perú. En esa década se oficializó el quechua y se empezó a implementar la educación intercultural bilingüe desde el Estado en el marco de una reforma educativa y de movimientos sociales que lucharon por una reforma agraria y por el empoderamiento económico de los campesinos. No obstante, ambas medidas fueron impulsadas básicamente por intelectuales y lingüistas de la capital. Al decir de Hornberger (1995), en aquel contexto de políticas de arriba hacia abajo, la población quechua hablante fue el grupo de interés invisible.

Volviendo sobre lo anterior, es importante señalar que los maestros de aula están influenciados por las ideologías de los expertos en quechua y que muchos de estos expertos también han sido maestros antes de adquirir mayor autoridad sobre los asuntos relacionados con la lengua. Los maestros pueden eventualmente convertirse en expertos cuando obtienen títulos, dominan el registro de los expertos y aprenden a ser como ellos. Dado que la experticia es algo que la gente hace en lugar de algo que la gente tiene, esta se alcanza 
a través de prácticas lingüísticas en el marco de las prácticas sociales (Carr, 2010). Es precisamente la perspectiva etnográfica la que tiene el potencial de capturar estos matices sobre los procesos implicados en la configuración de las prácticas sociales.

Desde un posicionamiento que les otorga mayores recursos económicos y simbólicos, este sector de hablantes de quechua empezó a construir una distinción entre ellos y los otros, a partir de una serie de ideologías lingüísticas. Así, por un lado, alimentan la idea de que el verdadero hablante de quechua es aquel que domina un quechua estandarizado que no está influido por el español, que sabe escribir con el alfabeto estándar y que tiene una consciencia gramatical explícita. Por otro lado, también refuerzan una ideología de la autenticidad, para la cual hablar quechua encarna una identidad "quechua" ligada a una cultura ancestral (ver más en Zavala, 2019). Para el propósito de este artículo, y por falta de espacio, me concentraré en el primer aspecto.

Lo que pasaré a analizar se enmarca en el fenómeno de la normatividad o de los regímenes de normatividad como lugar principal del carácter político del lenguaje. Mostraré cómo una entidad con autoridad o un sujeto de escucha (Flores y Rosa, 2015) impone una norma construida para legitimarse frente a otros y ejercer poder. En este caso, sin embargo, esta entidad (o este sujeto de escucha) está representada por una comunidad de hablantes de quechua que han sido cooptados por el Estado y el discurso oficial en los procesos de política lingüística. En alineación con los pilares teóricos de la glotopolítica, este caso que presento muestra cómo las representaciones en torno al quechua se negocian y disputan en los límites entre políticas institucionales y prácticas cotidianas. 
Estas controversias en torno a prácticas normativas entre los propios miembros de comunidades minorizadas han sido estudiadas discursivamente, históricamente pero también etnográficamente en diversos contextos latinoamericanos (ver King, 2001 y Wroblewski, 2012 en relación a los kichwas en Ecuador; Guerrettaz, 2020, en relación a los mayas en México; y Lagos, Espinoza \& Rojas, 2013, en relación a los mapuches en Chile) y en otras latitudes (ver Urla, 2015 para el caso del vasco; Jaffe, 1999 para el del corso; y O’Rourke y Ramallo, 2013, para el del gallego, entre otros). En todos los casos, la etnografía ha servido, no solo para desarrollar un análisis más orientado a los actores, sino también para dar cuenta de la forma en que el significado se va construyendo siempre de forma situada, en el marco de acciones y como parte de procesos.

\subsection{La opción etnográfica y lo que nos permite entender}

Desde una etnografía multisituada (Marcus, 1998), desarrollada en algunas regiones del sur andino y también en Lima, me topé con una multiplicidad de textos que me fueron revelando lo expuesto anteriormente. Esto no significa, sin embargo, que siempre las etnografías empiecen con análisis textuales pues, a veces, se puede llegar a los textos luego de observar alguna práctica o de conversar con alguien sobre algún tema.

En relación a los textos, revisé manuales escolares para la enseñanza del quechua, tanto a nivel de la educación básica como de cursos regulares para adultos, y pude constatar que se colocaba mucho énfasis en la escritura 
estandarizada pero también en la gramática y el conocimiento metalingüístico. Uno de los manuales para adultos (que no necesariamente se estaban formando como profesores) en la región de Apurímac, elaborado por expertos de quechua que trabajaban en una ONG de la región, señalaba las siguientes consignas (entre muchas otras): "Extrae palabras largas del cuento que leíste y separa la raíz y los sufijos", "Identifica la frase nominal en las siguientes oraciones", "Leemos y completamos en los puntos suspensivos la vocal o semivocal según corresponde" (Zavala, et al., 2014). Si bien desde mi formación de lingüista esto no me resultaba muy extrańo, el contacto con otros textos me ayudó a tomar distancia y buscar explicaciones para este hecho que, a primera vista, podía resultar un poco obvio y banal.

Más allá de estos textos educativos, en esta misma región, encontré copias de periódicos locales donde un grupo de expertos había publicado breves notas sobre el quechua algunos años antes, lo que sí me pareció un poco más "extraño" debido al contexto donde aparecían. A pesar de que los títulos de estas notas incluían frases como: "Aprendamos a leer y escribir en quechua" o “'Te gustaría aprender quechua?”, el contenido de las notas siempre era metalingüístico. Se incluyó el título "fonemas del quechua" con el listado de fonemas oclusivos, fricativos, nasales, laterales, vibrantes y semiconsonantes; o la clasificación "los sufijos flexivos de caso" con sus diferentes tipos (nominativo, genitivo y acusativo). Estas notas aparecían como parte de noticias locales sobre crímenes o anuncios publicitarios de ropa masculina.

Cuatro años después, ya en Lima, tuve la oportunidad de estudiar el proceso de evaluación del dominio de la lengua originaria por parte de los 
docentes como requisito para enseñar en las escuelas de Educación Intercultural Bilingüe y obtener una bonificación económica. Estas evaluaciones, construidas por expertos quechuas que trabajaban en el Ministerio de Educación (y algunos de los cuales procedían de la región de Apurímac), solo permitían el uso de ciertas características léxicas, estructurales y discursivas vinculadas a la lengua indígena estandarizada y definida políticamente, y terminaban borrando (Irvine y Gal, 2000) las prácticas lingüísticas de muchos hablantes bilingües, especialmente de los jóvenes. Por ejemplo, en las rúbricas de la evaluación, se pedía a los profesores que produjeran un quechua normalizado evitando el uso de "léxico innecesario" del español, órdenes sintácticos que pudieran tener una "influencia del español”, formas léxicas que no son de su "propia” lengua y patrones de entonación que no se consideran "auténticos". Esta manera de evaluar a los docentes estaba bastante naturalizada entre los evaluadores expertos de esa lengua, para quienes el quechua estandarizado era equivalente al "quechua" a secas. El contraste entre estas rúbricas y los otros textos recogidos me ayudaron a ir tomando distancia sobre la práctica social implicada.

Todos estos ejemplos (las consignas en manuales escolares, las notas en periódicos y las rúbricas de las pruebas para evaluar el dominio del quechua), entre otros más, fueron revelando que estos textos se inscriben en prácticas sociales a partir de las cuales se legitima solo un tipo de hablante o aprendiz de quechua: aquel que sabe escribirlo con el alfabeto estandarizado, conoce la gramática de forma explícita y lo habla desde un registro estándar que separa los recursos asociados al quechua y al español de forma estricta. 
Ahora bien, esta información de tipo textual pudo ser complementada con observaciones de algunos procesos donde se utilizaban los textos y con conversaciones con algunos sujetos involucrados. Así, fui dándome cuenta de los procesos de producción textual pero, también, de la forma en que los textos eran interpretados por aquellos que los consumían, todo lo cual me ayudó a ir explicando mejor el fenómeno.

Estas observaciones y conversaciones (o entrevistas) se complementaron entre sí y, a su vez, complementaron el análisis textual. Las observaciones de índole participante y no participante se enriquecen mutuamente cuando una se apoya en la otra, pues se observa para participar y se participa para observar (Guber, 2001, Brice Heath \& Street, 2008). Sin embargo, si bien se observa mejor cuando se participa, una participación muy intensa siempre implica el riesgo de que el investigador pierda distancia y olvide su papel para entender la conducta de la gente. A partir de las observaciones de aula de los cursos para adultos (donde participé muchas veces como alumna) pude darme cuenta de la forma en que los expertos del quechua enfatizaban el alfabeto estándar y los términos gramaticales para construir una distinción con sus estudiantes, muchos de los cuales conocían ya la lengua y eran bilingües con diferentes trayectorias. Participar en los cursos como alumna (y poder interactuar con otros alumnos) me permitió situarme en ese rol, medir un punto de vista "más interno" y percibir algunos matices que podían perderse de vista desde una mirada más distante. Además, en el caso específico de materiales educativos, es fundamental observar cómo estos se usan en el aula, pues los maestros pueden no hacer uso de ellos o 
utilizarlos de diversas maneras. También pude participar en otros tipos de eventos, donde asumí diversos roles.

El contraste entre las observaciones y lo que emerge en las entrevistas es importante, pues muchas veces las personas no terminan haciendo lo que dicen que hacen a la hora de ser entrevistadas. Así, por ejemplo, cuando les pregunté a los expertos sobre su propuesta pedagógica para enseñar quechua, varios solían mencionar el enfoque comunicativo con bastante solvencia, mostrando una contradicción con su enseñanza (ver casos similares en Jaffe, 2015, sobre el corso; Avineri, 2014, sobre el Yiddish; y Guerrettaz, 2020, sobre el maya). Esto muestra que las "generalizaciones de los miembros" (Scollon \& Scollon, 1994) que emergen en las entrevistas son parciales, pues las personas no suelen ser conscientes de algunos aspectos significativos de sus propias prácticas sociales. Así entonces, resulta fundamental que lo que las personas dicen se complemente con observaciones de lo que hacen en diferentes actividades y se anote aquello que está fuera de la conciencia del participante.

Asimismo, las observaciones del proceso de evaluación del dominio de la lengua originaria también me ayudaron a notar cómo, durante las calificaciones de las pruebas, los evaluadores estaban enfocados en ser muy estrictos con el puntaje que otorgaban a los maestros evaluados y solían bajarles puntos, lo cual pudo ratificar el análisis textual. En cuanto a los periódicos con notas en quechua, no pude conversar con los actores sociales involucrados, pues ya me encontraba en otro lugar cuando tuve acceso a ellos. Esta conversación hubiera sido muy útil para rastrear el proceso de 
producción y entender el marco más amplio de las prácticas en las que se publicaron estas notas. Me limité entonces a establecer conexiones entre este material y los otros que pude recolectar.

Otro tipo de observaciones no solo me permitieron ir reconstruyendo las prácticas, sino también ir percibiendo el sentir de los actores sociales y la forma en que estos consumían las ideologías dominantes. En una oportunidad, por ejemplo, pude presenciar una reunión en la municipalidad de la ciudad de Andahuaylas en Apurímac, liderada por expertos del quechua, donde diversos tipos de actores estaban discutiendo el futuro de la lengua en la región. Cuando la reunión terminó, la gente comenzó a dispersarse y pude escuchar el comentario que emitió una funcionaria municipal ante algunos expertos:

Yo veo que en el alfabeto quechua o en la escritura se viene forzando muchos términos, porque el quechua a mi modo de ver no se debe hacer tan difícil; si es un instrumento de comunicación, debemos comunicarnos con naturalidad, con espontaneidad, no importa si mezclamos el quechua y el castellano, pero de a pocas iremos avanzando. Pero, sin embargo, a veces, somos criticados, si utilizamos mal el quechua o mal algunos términos (...) cuando uno quiere estudiar o quieres practicar la escritura, se te hace tan difícil porque los estudios pues lo han forzado, me parece sí, por eso decía, discúlpenme si me equivoco, están forzando y están haciendo más difícil de lo que se piensa el quechua.

Si bien la funcionaria no lo hace explícito, en este extracto, que emergió fuera de una entrevista formal y como parte de un evento observado, revela que es consciente de que un grupo que estaría "forzando" la propuesta del desarrollo de la lengua en la región y de que los otros, como ella, se sienten "criticados" 
y finalmente excluidos. Se podría ańadir mucho más sobre este extracto, pero un análisis textual más extenso sobrepasa el objetivo de este trabajo.

En contraste con las observaciones, las conversaciones informales y entrevistas en profundidad con diversos actores sirven para entender el sentido que las prácticas sociales tienen para ellos. Esta reflexión de los actores sobre sus propias prácticas sociales es fundamental y solo se consigue entrevistando. En el caso del fenómeno analizado, no solo me interesaban las reflexiones de los propios expertos, sino sobre todo las de aquellos que consumían esta representación construida por los primeros. Si bien muchos expertos no eran totalmente conscientes de la forma en que enseñaban o evaluaban el dominio de la lengua originaria por parte de los maestros (y por eso era necesario mirar los textos y las prácticas), las entrevistas a los que consumían las ideologías lingüísticas de los expertos fueron clave para entender la recepción de este discurso.

Ahora bien, las conversaciones con las personas no solo deben enmarcarse en entrevistas formales grabadas y agendadas con anticipación, pues muchas veces los mejores datos emergen en los márgenes de lo que se intenta grabar de modo más sistemático (Bucholtz y Hall 2008). Tal es el caso de otra conversación que tuve con un profesor de quechua de una universidad en Andahuaylas (Apurímac) cuando fui a observar su clase. Si bien mi meta era esa, la conversación que emergió de modo informal minutos antes de que el profesor empezara su práctica y la anécdota que me contó me revelaron la forma en que el discurso de los expertos estaba "incapacitando" a muchas personas vinculadas con el quechua. Al decir 
de Blommaert y Jie (2010), "Las anécdotas son los diamantes en bruto en las entrevistas de campo".

Profesor: Llevé quechua en la universidad cuando estudiaba derecho y obtuve una nota de 13 promedio, "pucha", dije, "no, no, no puede ser, ¿cómo? hablo quechua yo puedo hablar el quechua mejor que el castellano y ¡̨cómo 13?” y el profesor, pues, manejaba todo, ahí me di cuenta que no sabía quechua.

Virginia: ¿Cómo no iba a saber si era de lengua materna quechua?

Profesor: Hablaba pero no podía escribir.

Virginia: Pero eso no significa no saber, solo significa no saber escribir.

Profesor: Para mi autoconcepto no sabía quechua.

El caso de este profesor es interesante pues se trata de una persona que está en proceso de convertirse en experto (en ese momento empezaba a enseñar quechua en una universidad y también trabajaba en una ONG elaborando materiales educativos en esta lengua), pero que recuerda el momento en que se matriculó en quechua como alumno universitario y estaba subordinado al discurso de los expertos (la frase "El profesor, pues, manejaba todo' hace alusión a la forma de actuar del experto). Sin embargo, no es plenamente consciente de este discurso y lo reproduce sin cuestionarlo. También es importante llamar la atención sobre mi participación en la conversación, pues yo desafío su respuesta a partir de dos pedidos de enmienda bastante 
categóricos (“¿Cómo no iba a saber si era de lengua materna quechua?” y "pero eso no significa no saber, solo significa no saber escribir”) y, a pesar de esto, él sigue sin cuestionar su perspectiva. Si bien mi participación puede percibirse como un poco "entrometida" para los parámetros de algunos paradigmas cualitativos, las respuestas del profesor me sirvieron para darme cuenta de cuán enraizado está el discurso analizado. Además, este ejemplo muestra las contradicciones y dilemas de una persona que está en el proceso de convertirse en experto, pero que todavía arrastra los efectos de su formación. Una de las características del trabajo etnográfico es precisamente que no simplifica la complejidad de las observaciones y de las entrevistas desde una perspectiva positivista de la investigación (Blommaert y Jie, 2010). En lugar de intentar aprehender la realidad desde lógicas lineales, dicotómicas y de causa efecto, la etnografía opta por hacerlo en términos más rizomáticos, donde la multiplicidad, impredecibilidad y complejidad resultan centrales.

Los datos recogidos de conversaciones con diferentes actores sociales pueden incluso ser más breves y menos estructurados. Así por ejemplo, durante un tiempo prolongado, las conversaciones informales con una multiplicidad de personas en diversos contextos y tiempos (taxistas, mozos en restaurantes, gente en la calle en general) me facilitaron darme cuenta de que el quechua, y sobre todo la escritura de la lengua, se percibía como "difícil". Esta opinión salió de forma recurrente una y otra vez, y pudo complementar las interacciones más prolongadas que tuve con otras personas en relación a sentirse excluidas del proyecto de desarrollo del quechua. Si bien podrían percibirse como sueltos y poco sistemáticos, tales datos puntuales, 
recogidos en una variedad de circunstancias (y anotados en un cuaderno), sirven eventualmente para armar el rompecabezas final y conseguir más densidad en el argumento. Como señala Blommaert y Jie (2010), la etnografía también requiere "recolectar basura" a lo largo de un tiempo prolongado, en el sentido de recoger fragmentos de información que, a primera vista, no parecieran ser tan necesarios para la investigación.

Todo el recuento anterior muestra que el análisis textual siempre debe enmarcarse en abordar las prácticas sociales de las que son parte, pues son las prácticas sociales las que finalmente nos interesa entender. Más allá del apoyo en un enfoque histórico, esto también implica una mirada etnográfica que, a su vez, supone observar las prácticas y recoger el sentido que los actores involucrados les dan a las mismas. La etnografía constituye una investigación de tipo inductivo y recursivo que supone ir integrando los fragmentos dispersos e ir haciendo conexiones entre pedacitos de información recogidos en diferentes niveles, tiempos y lugares. En un inicio esto puede ser frustrante, pero se va encontrando orden en el caos.

\subsection{Los jóvenes y la reacción a los expertos}

Si bien no voy a dedicarle mucho espacio a esta sección, sí me parece importante señalar que el discurso de los expertos ha comenzado a generar resistencias desde la última década y que la mirada de la etnografía es útil para entender estas dinámicas que se vienen desarrollando en el país. Dentro de los actuales procesos de globalización, una comunidad de práctica 
de jóvenes bilingües en zonas urbanas (sobre todo en Lima) se encuentra contrarrestando estas acciones de guardianía de los expertos y colocando la agencia juvenil en el centro de las iniciativas de las políticas lingüísticas. Desde una mirada glotopolítica, se trata de discursos disidentes, inseparables de la generación de subjetividades alternativas e imbricados en lo político desde los márgenes de los discursos oficiales (Arnoux \& Bentivegna 2019).

La vida social en el siglo XXI ha producido un nuevo escenario sociolingüístico en el Perú, en el que los repertorios, experiencias y trayectorias de muchas personas en relación al quechua no encajan dentro de las categorías fijas ni en la vigilancia de las fronteras lingüísticas y étnicas que los expertos que ejercen poder han reproducido en torno a la categoría de lo quechua y de los hablantes de quechua. En el Perú, son los jóvenes, especialmente los universitarios quechuas, quienes están construyendo una versión diferente de la lengua y de lo quechua. Este fenómeno debe enmarcarse en un escenario en el que esos jóvenes tienen mucho más acceso a la educación superior y utilizan los nuevos medios de comunicación con regularidad, lo que les ofrece grandes posibilidades de reflexión crítica y comunicación con un público más amplio, que comparte preocupaciones similares.

Mi atención a este fenómeno surgió en el año 2014 cuando vi en Internet un video de un joven que cantaba rap en quechua. Lo contacté por messenger y pude conversar con él. Luego, me di cuenta no solo de que había otros que cantaban hip hop, reggaeton, trap, pop o rock clásico en quechua, sino de que se estaba formando un movimiento de jóvenes activistas que están cambiando las representaciones en torno al quechua y lo quechua. Este movimiento 
también incluye a blogueros, comunicadores, poetas, editores de revistas en quechua y profesores que dictan la lengua en diferentes lugares de la ciudad de Lima. Con el tiempo, pude constatar cómo se estaba conformando una comunidad de práctica de "difusores" de la lengua (así se han comenzado a llamar ellos mismos) y cómo empiezan a diferenciarse de los expertos. En palabras de un joven cusqueño que dobla películas de Disney, estos expertos serían "un grupo de quechua hablantes antiguos que mitifican un poco el tema del quechua y quieren ser demasiado exquisitos pero, al mismo tiempo, en ese intento de generar su exquisitez, caen en ciertas torpezas".

El trabajo etnográfico con estos jóvenes ha implicado el análisis de una variedad de tipos de textos: letras de canciones de rap, manuales para la enseñanza del quechua elaborados por ellos mismos, posts e interacciones en Facebook y otras plataformas digitales, diversos tipos de escritos publicados en revistas, entre otros; pero siempre articulados con múltiples entrevistas y repetidas observaciones. En las entrevistas (que usualmente son más de una por entrevistado) he podido recoger las ideologías lingüísticas que se están reconfigurando desde esta comunidad de práctica. Asimismo, mis observaciones de una variedad de prácticas me han ayudado a acceder a “escenarios glotopolíticos” (Castro y Del Valle, 2019) donde los jóvenes negocian representaciones y subjetividades con relación a los recursos lingüísticos. Tal es el caso de eventos culturales, donde se reúnen para difundir el quechua, o clases de quechua dictadas por ellos mismos en diferentes lugares de Lima. A partir de las entrevistas y de las observaciones he podido ir entendiendo la lógica de una política lingüística mucho más incluyente, 
que busca legitimar a una mayor cantidad de hablantes en el marco de un paradigma pos-tradicional de revitalización lingüística (Pietikäinen, et al., 2016, ver Zavala, 2019 para un mayor desarrollo).

Quisiera terminar esta sección con dos puntos que no desarrollé cuando me refería a los expertos y que también definen la etnografía. El primero es de carácter metodológico y se refiere al tipo de entrevistas que se pueden hacer. Si bien las mismas capturan el sentido que les dan los participantes a sus prácticas sociales, esta información puede a veces resultar muy general (Scollon y Scollon, 1994). Por eso, en el caso del fenómeno que estoy presentando, las entrevistas a los jóvenes fueron complementadas con "historias de vida" de participantes focales. Los testimonios de vida, historias orales o estudios de caso de participantes específicos contribuyen a capturar la riqueza de la experiencia individual de los miembros, en contraste con la práctica social generalizada. Las múltiples conversaciones con varios jóvenes revelaron cómo las ideas y sentimientos sobre el lenguaje están articulados con sus vidas sociales, biografías e historias; y con los discursos disidentes que colectivos juveniles despliegan actualmente con relación a la lengua originaria. Más allá del contexto histórico general, la historia personal de los sujetos de la investigación también ayuda a comprender los procesos estudiados y enriquece los datos etnográficos.

El segundo punto se refiere a la importancia de la reflexividad y a explicitar el posicionamiento del investigador en todo proyecto etnográfico. En lugar de esconder mis ideas en relación a las lenguas originarias y mostrarme como imparcial, siempre he intentado investigar con los suje- 
tos involucrados a partir de métodos más abiertos, interactivos y dialógicos (Cameron, et al., 1992). En ese sentido, no solo les he mostrado mis resultados en diferentes momentos de la investigación con el fin de recibir críticas, sino que he tratado de compartir con ellos mis marcos teóricos y las ideas que voy procesando conforme avanza el recojo de mis datos. Esto no solo mejora nuestra comprensión de lo que observamos (como vimos en el extracto anterior con el profesor de quechua), sino que permite compartir el conocimiento académico y, en muchos casos, empoderar a algunos actores sociales participantes de la política lingüística, tal como ha ocurrido con muchos jóvenes con los que he trabajado.

\section{Reflexiones finales}

El estudio social del lenguaje se ha potenciado mucho en las últimas décadas, gracias a diversas corrientes que están optando por articular una mirada interdisciplinaria, crítica, histórica y etnográfica. Aunque la etnografía puede ser difícil de implementar cuando se trata de datos históricos, sí puede acompañar a una diversidad de investigaciones sobre las representaciones del lenguaje en entornos glotopolíticos contemporáneos. Desde los pilares de la glotopolítica, sabemos que la lengua constituye un terreno para la construcción de relaciones de diferencia y desigualdad. No obstante, considero que "el análisis discursivo, la lectura ideológica y la historificación" (Arnoux y Del Valle, 2010, 19) necesitan de la etnografía para alcanzar un mayor poder explicativo de los fenómenos abordados. La etnografía ayuda 
precisamente a visibilizar a las personas como actores sociales y las fronteras como productos de la acción social (Heller, 2020. Los textos serían solo expresiones de estos procesos.

La glotopolítica y los estudios críticos del lenguaje nos permiten combinar la práctica, la ideología y la economía política en el estudio de las lenguas originarias, el bilingüismo y las políticas lingüísticas en nuestra región. Si vinculamos la lengua con la economía política podemos explicar los procesos de diferenciación y desigualdad social en un contexto en el que algunas personas pueden convertir o intercambiar eficazmente sus recursos lingüísticos en otras formas de capital, mientras que otras no. Este caso de los expertos en quechua muestra cómo ciertas formas y prácticas verbales de la lengua indexan posiciones de clase que están conectadas con la propiedad y el control sobre los medios de producción (Irvine 1989). El conocimiento de la literacidad, la gramática y el quechua estandarizado ha adquirido valor, puede ser intercambiado por bienes materiales y ahora beneficia a un grupo específico de hablantes de quechua, que se erigen como los que "saben quechua" para mantener sus privilegios. Por lo tanto, no basta con posicionar a las lenguas como medios de comunicación o marcadores de identidad, ya que también constituyen competencias valoradas en los mercados de trabajo. De hecho, estas batallas por los significados en torno al quechua no solo reflejan una economía de las ideas sino también privilegios simbólicos y materiales, que están vinculados a empleos, salarios, oportunidades para enseñar la lengua, acceso a publicaciones, entre otros recursos. El enfoque 
etnográfico se suma al enfoque histórico para ayudarnos a entender mejor los procesos implicados en este fenómeno.

$\mathrm{Al}$ igual que el análisis del discurso con perspectiva crítica, la etnografía también constituye un emprendimiento crítico y contrahegemónico (Blommaert y Jie, 2010), que ayuda a deconstruir representaciones y a asumirlas como problemas y no como hechos. Desde una mirada interna a los actores sociales involucrados, enriquece el estudio de la historia de las ideas lingüísticas otorgándole un mayor poder explicativo. Si la sociolingüística crítica y la antropología lingüística del norte global tienen un largo recorrido abordando el lenguaje con una perspectiva etnográfica, creo que la glotopolítica podría sumarse a estos desarrollos.

\section{REFERENCIAS BiBLIOGRÁFICAS}

Arnoux, Elvira Narvaja de; José del Valle. "Las representaciones ideológicas del lenguaje. Discurso político y panhispanismo". En: Spanish in Context, 7 (1), 2010, 1-24.

Arnoux, Elvira Narvaja de; Susana Nothstein (eds.). Temas de glotopolitica: Integración regional sudamericana y panhispanismo. Buenos Aires: Biblos, 2013.

Arnoux, Elvira Narvaja de; Diego Bentivegna. "Introducción al dossier 'lenguas y discursos disidentes"'. Heterotopias, 2 (4), 2019, 1-9.

Avineri, Netta. 2014. "Yidish endangerment as phenomenological reality and discursive strategy: Crossing into the pastand crossing out the present". In: Language and Communication, 38, 18-32. 
Blommaert, Jan. "Rights in places: Comments on linguistic rights and wrongs". In: Freeland, Jane \& Patrick, Donna (eds.). Language rights and language survival. London: Routledge, 2004, 55-65.

Blommaert, Jan. "Language Ideology". In: Keith Brown (ed.). Encyclopedia of Language \& Linguistics. Oxford: Elsevier, Second Edition, volumen 6, 2006, 510-522.

Blommaert Jan. \& Jie, Dong. Ethnographic fieldwork. A beginner's guide. Bristol: Multilingual Matters, 2010.

Bourdieu, Pierre. Razones prácticas. Sobre la teoría de la acción. Barcelona: Anagrama, 1997.

Brice Heath, Shirley; Street, Brian. Ethnography. Columbia: Teachers College, 2008.

Bucholtz, Mary; Kira Hall. "Todo lo anterior: nuevas coaliciones en la lingüística sociocultural”. In: Autor et al. (ed.), 2020 [2008].

Cameron, Deborah; Elizabeth Frazer; Penelope Harvey, M.B.H. Rampton y Kay Richardson (ed.). Researching language. Issues of power and method. Londres: Routledge, 1992.

Castro Picón, Natalia; José Del Valle. "I have a scream": Enunciaciones disidentes en torno al 15M en España. In: Heterotopias, 2 (4), 2019, 1-15.

Carr, Summerson. "Enactments of expertise". In: Annual Review of Anthropology, 39, 2010, 17-32.

Del Percio, Alfonso; Flubacher, Mi-Cha; Duchene, Alexander. "Language and political economy”. In: Ofelia García, Nelson Flores \& Massimiliano Spotti (Eds.). The Oxford Handbook of Language and Society. Oxford: Oxford University Press, 2017, 55-75.

Del Valle, José. "Glotopolítica y teoría del lenguaje. La perspectiva glotopolítica y la Normatividad”. In: Anuario de Glotopolítica, 1, 2017. 
Flores, Nelson; Rosa, Jonathan. "Deshaciendo la noción de adecuación: Ideologías lingüístico-raciales y diversidad lingüística en educación”. In: Autor et al. (ed.), 2020 [2015].

Guber, Rosana. La etnografía. In: Método, campo y reflexividad. Buenos Aires: Norma, 2001.

Guerrettaz, Anne Marie. “¿A quiénes les pertenece el maya yucateco? Revitalización y políticas lingüísticas en Yucatán, México”. In: Mercedes Niño-Murcia; Virginia Zavala; Susana de los Heros (eds.); Hacia una sociolingüistica crítica: Desarrollos y debates. Lima: Instituto de Estudios Peruanos, 2020, 267-303

Heller, Monica. "Hacia un enfoque social del bilingüismo". In: Mercedes NiñoMurcia, Virginia Zavala y Susana de los Heros (eds.); Hacia una sociolingüística critica: Desarrollos y debates. Lima: Instituto de Estudios Peruanos, 2020.

Heller, Monica, Pietikainen, Sari \& Pujolar, Joan. Critical sociolinguistic research methods. Studying language issues that matter. Nueva York: Routledge, 2018.

Hornberger, Nancy. "Five vowels or three? Linguistics and politics in Quechua language planning in Perú". In: James Tollefson (ed.). Power and inequality in language education. Cambridge, UK: Cambridge University Press, 1995, 187-205.

Irvine, Judith. "When talk isn't cheap: language and political economy". In: American Ethnologist, 6 (2), 1989, 248-267.

Irvine, Judith, \& Gal, Susan. "Language ideology and linguistic differentiation". En: Paul Kroskrity (Ed.). In: Regimes of language, ideologies, polities and identities. Nuevo Mexico: School of American Research Press, 2000, 35-84.

Jaffe, Alexandra. Ideologies in action: Language politics on Corsica. Nueva York: Walter de Gruyter, 1999.

Jaffe, Alexandra. "Defining the new speaker: Theoretical perspectives and learner trajectories". International Journal of the Sociology of Language, 231, 21-44. 
King, Kendall. Language revitalization processes and prospects. Nueva York: Multilingua Matters, 2001.

Lagos, Cristian; Espinoza, Marco; Rojas Darío. "Mapudungun according to its speakers: Mapuche intellectuals and the influence of standard language ideology”. In: Current Issues in Language Planning, 14 (3), 2013, 1-16.

Lave, Jean; Etienne Wenger. Situated learning: Peripheral legitimate participation. Cambridge, UK: Cambridge University Press, 1991.

Lillis, Theresa. "Ethnography as method, methodology, and 'Deep Theorizing'. Closing the gap between text and context in academic writing research". In: Written Communication, 25 (3), 2008, 353-388.

Marcus, George. "Ethnography in/of the world system: the emergence of multisited ethnography". In: Ethnography through thick and thin. Princeton: Princeton University Press, 1998, 79-104.

O’Rourke, Bernadette; Ramallo, Fernando. "Competing ideologies of linguistic authority amongst 'new speakers' in contemporary Galicia". In: Language in Society, 42(3), 2013, 287-305.

Pennycook, Alastair. Language as a local practice. Nueva York: Routledge, 2010.

Pietikäinen, Sari, Kelly-Holmes, Helen, Jaffe, Alexandra \& Coupland, Nicholas. Sociolinguistics from the Periphery. Small Languages in New Circumstances. Cambridge: Cambridge University Press, 2016.

Scollon, Ron \& Suzanne W. Scollon. Intercultural communication. A discourse approach. Filadelfia: Blackwell, 1994.

Scollon, Ron \& Suzanne W. Scollon. Nexus analysis: discourse and the emerging internet. Nueva York: Routledge, 2004.

Urla, Jacqueline. Reclaiming Basque. Language, nation and cultural activism. Nevada: University of Nevada Press, 2015. 
Wroblewski, Michael. "Amazonian Kichwa proper: ethnolinguistic domain in PanIndian Ecuador". In: Journal of Linguistic Anthropology, 22 (1), 2012, 64-86.

Zavala, Virginia. "Youth and the repoliticization of Quechua". In: Language, Culture and Society, 1(1), 2019, 59-82.

Zavala, Virginia. "Tactics of intersubjectivity and boundary construction in language policy: An Andean case”. In: Language, Identity and Education, 19 (2), 2020, 95-110.

Zavala, Virginia; Mujica, Luis; Córdova, Gavina; Ardito, Wilfredo. Qichwasimirayku Batallas por el quechua. Lima: Fondo Editorial de la Pontificia Universidad Católica del Perú, 2014. 